\title{
Pair-correlation kinetics and the reversible diffusion-controlled reaction
}

\author{
David Peak, David C. Greenlaw, and Louis A. Schick \\ Department of Physics, Union College, Schenectady, New York 12308
}

(Received 15 December 1989)

\begin{abstract}
It has long been known that the time course of a bimolecular reaction occurring in a condensed host depends on the behavior of the nonequilibrium pair-correlation function for reactant pairs. The classical analysis of such reactions has led to a kind of standard rule: The association rate constant for a diffusion-controlled reaction is $4 \pi D R$ and this rate constant produces the fastest possible kinetics. This result is only (approximately) true for the case of an irreversible reaction, however. Here, we reexamine this old problem, looking closely at the reversible case. We report a result that challenges the standard wisdom: When the reaction is highly reversible the relaxation of the related kinetics to equilibrium can be much faster than the model in which $4 \pi D R$ is the association rate constant. We suggest that our work provides a natural resolution to a well-known, long-standing controversy in the study of electrically active impurities in silicon grown by the Czochralski method.
\end{abstract}

The rates at which physical and chemical changes occur in condensed media are often determined by the mobilities of the individual members of the participating species. Such a situation has been termed "diffusion controlled." While the effects of diffusion on condensed phase reaction rates have been studied for many years, ${ }^{1,2}$ the diffusion-controlled reversible reaction-despite its ubiquity and importance - has only recently received any careful attention. ${ }^{3}$ Frequently, discussions of diffusioncontrolled reversible reactions have assumed that the bimolecular association rate could be represented with negligible error by the "standard" form $4 \pi D R$, where $D$ is a mutual diffusivity and $R$ an encounter distance for the appropriate reactants. In this paper, we discuss the limits of the validity of this assumption and argue that its use to infer values of diffusivities can lead to significant overestimates.

Though the work reported here has a much more general applicability, we were initially drawn to this problem by our interest in the well-known problem of the formation of electrically active oxygen clusters in Czochralskigrown silicon crystals. ${ }^{4}$ Succinctly stated, a considerable body of evidence now exists which implies that when such material is annealed at about $700 \mathrm{~K}$ (after quenching from the melt to room temperature), isolated impurity oxygen atoms become mobile and aggregate, producing, as a result, easily ionized donor states. Straightforward kinetics models developed to describe this phenomenon share a common failing: in order to quantitatively fit the kinetics data, interstitial oxygen atoms must be assigned mobilities greatly in excess of those measured by techniques independent of reaction. ${ }^{5}$

The essential features of the source of the discrepancy outlined above can be extracted from a study of the simplest association process, namely, dimerization, $A_{1}$ $+A_{1} \leftrightarrow A_{2}$, to which we now restrict our attention. We assume that the kinetics of the dimerization process is governed by a potential energy surface similar to that shown in the schematic sketch, Fig. 1. In a "diffusion- controlled" reaction $E_{D}>E_{+}$. Following the guidance of previous discussions, ${ }^{6}$ we postulate that the kinetics of this process can be described by

$$
\frac{d c_{1}}{d t}=-\frac{2 d c_{2}}{d t}=-k_{+}^{\prime}(t) c_{1}^{2}+2 k_{-} c_{2},
$$

where the $c$ 's are sample-average concentrations, $k_{+}{ }_{+}$is a time-dependent, bimolecular rate coefficient, and $k_{-}$is the dimer dissociation rate (see Fig. 1). The coefficient $k^{\prime}+$ depends on the probability that at any instant, two monomers will actually be separated by the critical distance $R$. (We ignore the crystal structure of the host here.) To determine just how $k^{\prime}$ depends on time we introduce the (coarse-grained) pair concentration $\rho(r, t)$. Specifically, $\rho$ is the concentration of $A_{1}-A_{1}$ pairs at time $t$ whose members are separated by a distance $r ; \rho$ is normalized so that its value for large separations approaches $c_{1}^{2}$. (Note that in this normalization pairs are double counted.) We take $V$ to be the volume defined by the re-

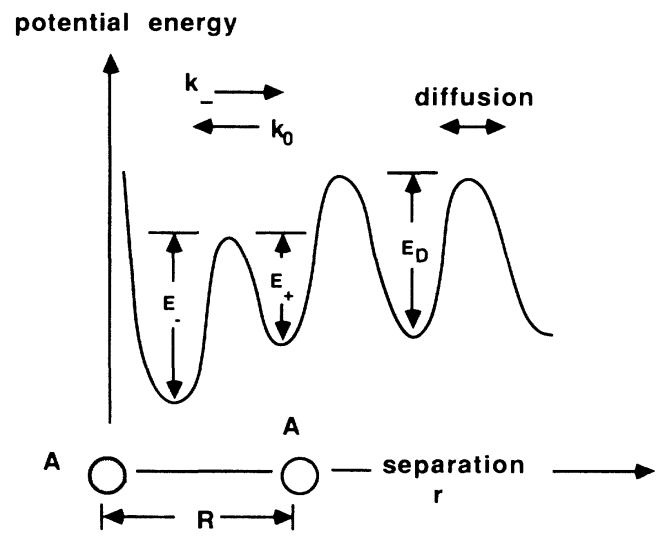

FIG. 1. Qualitative sketch of the potential energy of interaction between two monomers immersed in a dense host. 
action distance $R$. Then $V \rho(R, t)$ is (twice) the instantaneous concentration of monomer pairs with critical member separation. Since $k_{0}$ is the rate monomers cross the energy barrier $E_{+}, k_{0} V \rho(R, t)$ represents the rate at which monomers disappear due to reaction, or, in other words, $k_{+}^{\prime}(t)=k_{0} V \rho(R, t) / c_{1}^{2}$.

Clearly, the dimerization kinetics are governed by the behavior of the pair concentration. We expect that $\rho(r, t)$ will satisfy an equation of the type $\partial \rho(r, t) / \partial t$ $=D \nabla^{2} \rho(r, t)+\mathcal{R}(r, t)$, where $\mathcal{R}$ accounts for all reactive gains and losses of $A_{1}-A_{1}$ pairs with member separation $r$. These result whenever (a) either member of such a pair forms a dimer with a third monomer or (b) a dimermonomer pair of separation $r$ becomes a monomer triplet through the dissociation of the dimer. ${ }^{7}$ In addition to having to specify $\mathcal{R}$, determination of $\rho$ also requires specification of appropriate boundary and initial conditions. One boundary condition has already been assumed: $\rho \rightarrow c_{1}^{2}$ as $r$ becomes large. A second boundary condition can be obtained by considering the possible changes in the concentration $V \rho(R, t)$. Pairs of member separation $R$ can be lost by dimerization, can be gained by dimer dissociation, and can be either lost or gained by diffusion. Thus, the second required boundary condition is

$$
\begin{aligned}
\frac{\partial[V \rho(R, t)]}{\partial t}= & -k_{0} V \rho(R, t)+2 k_{-} c_{2}(t) \\
& +4 \pi D R^{2} \frac{\partial \rho(R, t)}{\partial r} .
\end{aligned}
$$

In the irreversible case, where $k_{\text {- }}$ vanishes, the left-hand side of (2) is often set equal to zero leading to the socalled "radiation boundary condition." For the reversible reaction, however, the full-time dependence of (2) is required. The reversible reaction kinetics are automatically rendered analytically intractable as a consequence.

It is convenient to rewrite the pair concentration $\rho$ in terms of the monomer-monomer (coarse-grained) paircorrelation function $h(r, t): \rho(r, t)=c_{1}^{2}[1+h(r, t)]$. Substitution of this form for $\rho$ into the diffusion-reaction equation of which it is a solution leads to

$$
\frac{\partial h(r, t)}{\partial t}=D \nabla^{2} h(r, t)+\mathcal{R}_{h}(r, t),
$$

where $\mathcal{R}_{h}$ depends on monomer triplet correlations as well as dimer-monomer correlations. We will focus on highly reversible reactions at low concentrations. We have explored different model expressions for $\mathcal{R}_{h}$, but have found that, for the dilute reactant case, this term contributes little to the kinetic behavior of the paircorrelation function. Its precise form is therefore irrelevant for our present discussion. Also, in the lowconcentration case the boundary condition (2) can be approximated by

$$
\begin{aligned}
\frac{\partial h(R, t)}{\partial t}= & -k_{0}[1+h(R, t)]+2 k_{-} c_{2} /\left(V c_{1}^{2}\right) \\
& +\left(4 \pi D R^{2} / V\right) \partial h(R, t) / \partial r .
\end{aligned}
$$

Equations (1), (3), and (4), then, along with the conditions $h \rightarrow 0$ as $r$ becomes large (no correlation among pair members at large separation), $h=0$ for all separations at $t=0 \quad$ (no initial correlation among monomer pair members), and values for $c_{1}(0)$ and $c_{2}(0)$, define a wellposed mathematical problem. Condition (4) is the key to obtaining appropriate pair correlations for the reversible, diffusion-controlled reaction; its incorporation is the essential difference between our analysis and all those that have preceded it.

Let us assume that the reaction is diffusion-controlled and that the monomers are initially uniformly randomly distributed. Suppose that the initial concentration of dimers is well below its equilibrium value at the temperature to which the sample is rapidly raised and fixed. Then some fraction of the monomer pairs with initial separation $R$ will quickly associate and $h(r, t)$ will become negative for $r$ near $R$. Later, both diffusion and dissociation will try to fill in this "correlation hole." In an irreversible reaction it is easy to show that the paircorrelation function approaches a steady approximate value, $h(r, t) \rightarrow-R / r$, as time goes on. In this case, $k_{0} V[1+h(R, t)]$ approximates the "diffusion-controlled" rate constant $4 \pi D R$. In the reversible case, however, the dissociation reaction keeps filling the correlation hole back in so that, after a period of being negative, $h$ eventually returns to zero for all $r$ as equilibrium is established. For this circumstance, $k_{0} V[1+h(R, t)]$ is always greater than $4 \pi D R$.

The time course of the "pair-correlation kinetics" described above is bounded from below by a fictitious, though often used, kinetics model. In this model, $k_{0} V[1+h(R, t)]$ is replaced by $4 \pi D R$ and $k_{-}$is replaced by an effective dissociation rate constant chosen to make the equilibrium state of these kinetics agree with that of the pair-correlation kinetics model. This latter model we call the "diffusion-controlled" kinetics model. In it the

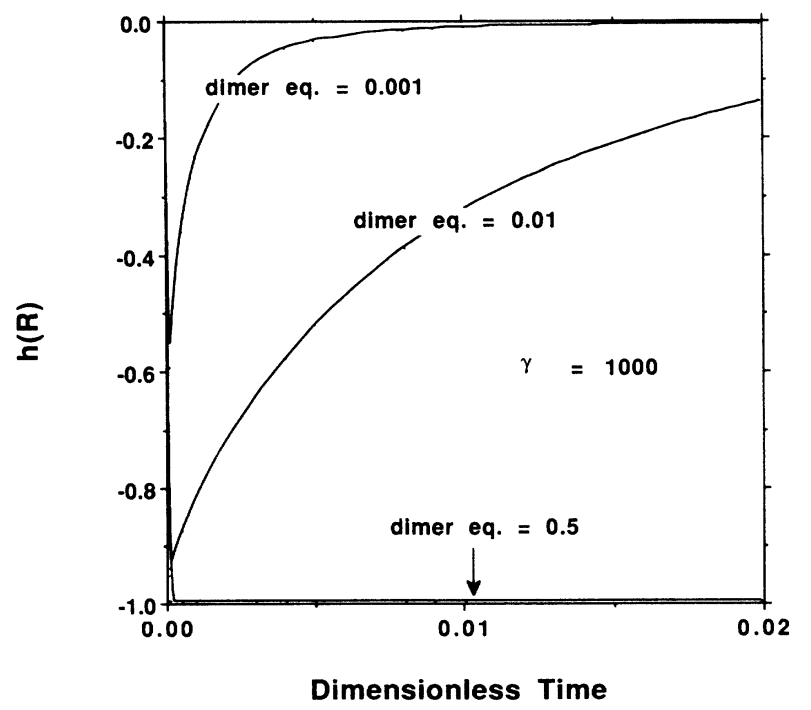

FIG. 2. The pair-correlation function for separation equal to the critical reaction distance. Time is measured in units of $2 / 4 \pi D R c_{0}$. "Dimer eq." is the equilibrium dimer concentration in units of $c_{0}$. 


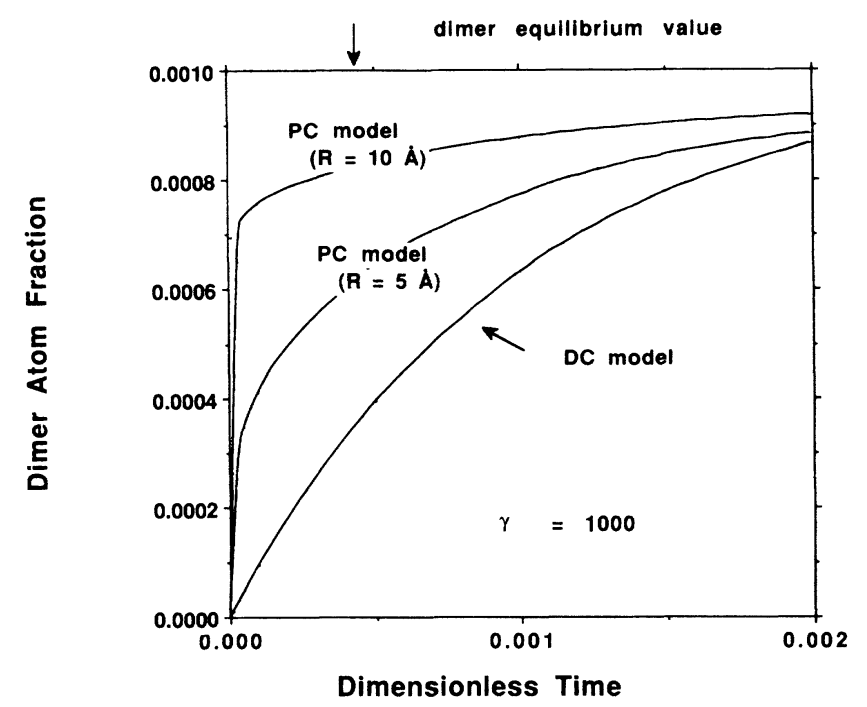

FIG. 3. Dimer concentration, measured in units of $c_{0}$, as a function of time (in units of $2 / 4 \pi D R c_{0}$ ). $P C$ corresponds to the pair-correlation model, $D C$ to the diffusion-controlled model.

effective dissociation rate constant $k_{-}^{\prime}$ is given by $k_{-}^{\prime}=k_{-} / \gamma$, where $\gamma$ is the ratio $k_{0} V / 4 \pi D R$ (assumed to be $\gg 1$ for a diffusion-controlled reaction). This model tacitly assumes that the pair correlations are always constrained to be in steady state. The diffusioncontrolled model relaxes toward equilibrium at a characteristic rate determined by both $4 \pi D R$ and $k_{-}^{\prime}$. Since both of these rate constants are lower than the corresponding rate coefficients of the pair-correlation model, the latter always approaches equilibrium more rapidly than the former.

We show in Figs. 2 and 3 the results of numerically integrating the pair-correlation model. We chose parametric values typical of thermal donor phenomena: oxygen interstitial diffusivity of $\left(0.2 \mathrm{~cm}^{2} / \mathrm{s}\right) \exp \left(-2.5 \mathrm{ev} / k_{B} T\right)$, temperature at $450^{\circ} \mathrm{C}$, total oxygen concentration, $c_{0}=10^{18} \mathrm{~cm}^{-3}$. Furthermore, we assumed highly diffusion-controlled conditions: $\gamma=1000$. In all cases, $c_{2}(0)$ was taken to be zero (only monomers in the initial state). Figure 2 demonstrates the behavior of the paircorrelation function for small separations; time is scaled in units of $4 \pi D R c_{0} / 2$. Each curve was calculated assuming $R=5 \AA$. The different cases shown represent different degrees of reversibility. Each time course corre-

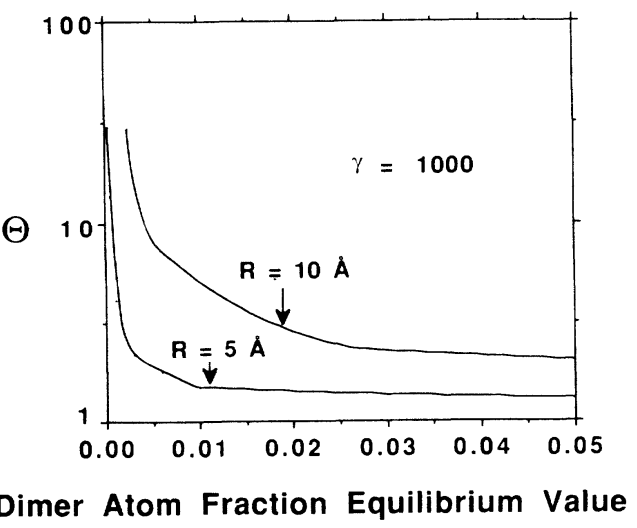

FIG. 4. Shows how the effective kinetics-based diffusivity of the diffusion-controlled model varies with the degree of reversibility of the reaction. "Theta" is the ratio of the effective to the actual diffusivities, $\Theta=D_{\text {eff }} / D$.

sponds to a different dimer equilibrium concentration measured in units of $c_{0}$. A dimer equilibrium value of 0.5 means that all monomers eventually wind up bound in dimers - the case of total irreversibility. For a totally irreversible reaction, $h(R, t)$ quickly becomes -1 and stays there. For more reversible cases, however, $h(R, t)$ goes less negative and eventually recovers to its starting value of zero.

Figure 3 shows the effect on the kinetics of the paircorrelation behavior described in Fig. 2. Here all examples evolve to the same dimer equilibrium concentration, namely, $0.001 c_{0}$. Two pair-correlation calculations, one with $R=5 \AA$, the other with $R=10 \AA$, are shown. Both rise toward equilibrium more rapidly than the corresponding diffusion-controlled calculation (which in these time units is independent of $R$ ).

Finally, we note that the diffusion-controlled kinetics can be fit to the pair-correlation kinetics by assuming an effective diffusivity, $D_{\text {eff }}=\Theta D$, where $\Theta$ is the fitting parameter. Figure 4 depicts how the fitting parameter varies with degree of reversibility. The point of the figure is that the more reversible the reaction the larger is the required $D_{\text {eff }}$ in order to fit the more realistic kinetics with the fictitious diffusion-controlled model. We see that for a reversible diffusion-controlled reaction, this effective, kinetics-based diffusivity can easily be an order of magnitude or more larger than the actual diffusivity appropriate to the system under study.
${ }^{1}$ M. von Smoluchowski, Phys. Z. 17, 585 (1916); Z. Phys. Chem. Leipzig 92, 129 (1917).

${ }^{2}$ For a partial listing of many other contributions to this field, see the extensive review by D. F. Calef and J. M. Deutch, Ann. Rev. Phys. Chem. 34, 493 (1983).

${ }^{3}$ See, e.g., M. A. Burschka, C. R. Doering, and D. ben-Daniel, Phys. Rev. Lett. 63, 700 (1989), and references therein.
${ }^{4}$ C. S. Fuller, J. W. Dietzenberger, N. B. Hannay, and E. Buehler, Phys. Rev. 96, 833 (1954); C. S. Fuller and R. A. Logan, J. Appl. Phys. 28, 1427 (1957); W. Kaiser and P. H. Keck, ibid. 28, 822 (1957); W. Kaiser, Phys. Rev. 105, 1751 (1957). See also reviews by J. R. Patel, in Semiconductor Silicon 1981, edited by H. R. Huff, R. J. Kriegler, and Y. Takeishi (Electrochemical Society, Pennington, NJ, 1981), p. 189; 
and by U. Gosele and T. Y. Tan, Appl. Phys. A28, 79 (1982).

${ }^{5}$ W. Kaiser, H. L. Frisch, and H. Reiss, Phys. Rev. 112, 1546 (1958); A. Ourmazd, W. Schroter, and A. Bourret, J. Appl. Phys. 56, 1670 (1984); J. T. Borenstein, D. Peak, and J. W. Corbett, J. Mater. Res. 1, 527 (1986).

${ }^{6}$ T. R. Waite, Phys. Rev. 107, 463 (1957).
${ }^{7}$ The form of the competitive reaction term $\mathcal{R}$ has been discussed in a number of places such as in Ref. 6, in D. Toussaint and F. Wilczek, J. Chem. Phys. 78, 2642 (1983), and in D. Peak, T. C. Werner, R. M. Dennin, Jr., and J. K. Baird, ibid. 79, 3328 (1983). 


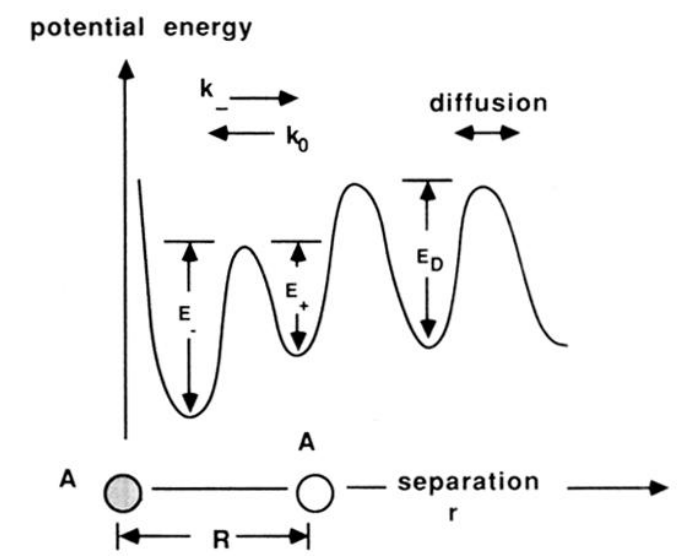

FIG. 1. Qualitative sketch of the potential energy of interaction between two monomers immersed in a dense host. 